\title{
Oncogenic Pathways in the Development of Oral Cancer
}

\section{Rui Amaral Mendes}

Department of Oral Medicine/Oncology and Clinical Pathology, Portuguese Catholic University, Portugal

Oral carcinogenesis is a molecular and histological multistage process featuring genetic and phenotypic markers for each stage, which involves enhanced function of several oncogenes and/or the deactivation of tumor suppressor genes, resulting in the loss of cell cycle checkpoints.

The progression towards malignancy includes sequential histopathological alterations ranging from hyperplasia through dysplasia to carcinoma in situ and invasive carcinoma, which are determined by the accumulation of a series of genetic events. In cellular carcinogenesis, various genes interact with each other, thus leading to multiple alterations that occur in a rather complex way and in different stages of progression of the disease [1].

Several signal transduction pathways have been reported to be altered in cancer, leading to dramatic changes in cell survival, cell proliferation, morphology, angiogenesis, longevity and other properties, which characterize cancer cells.

Epidermal Growth Factor Receptor (EGFR), one of the best studied oncogenes, plays an important role in the control of cellular proliferation, apoptosis, invasion, angiogenesis, and metastasis, as it works through the tyrosine kinase cascade. This receptor tyrosine kinase, also known as type I receptor tyrosine kinases or ErbB tyrosine kinase receptors, has many downstream signaling targets associated with carcinogenesis. Once phosphorylated, the receptor can signal via the MAPK, Akt, ERK, and Jak/STAT pathways.

Abnormality of EGFR gene and overexpression of the protein have been reported in various human tumors, with an abnormal amplification of the EGFR gene being reported in oral squamous cell carcinoma, although not limited to the final stages of the carcinogenic process. In fact, a low level of gene amplification also occurs at a significant frequency in epithelial dysplasia and carcinoma in situ, and, moreover, increased EGFR gene copies via amplification seems to play an important role in the development of invasive cancer [2].

Moreover, the expression of proliferation markers TGF- $\alpha$ and EGFR in cells of the oral epithelium presenting a spectrum of dysplastic changes revealed a serial upregulation both in terms of area and intensity of staining of TGF- $\alpha$ in the epithelial cells of oral precancerous lesions exhibiting features of dysplasia. Likewise, TGF- $\alpha$ expression has been reported higher than EGFR's in the proliferative pool of the oral epithelium in oral precancer lesions, thus suggesting that an initial upregulation of TGF- $\alpha$ was likely to exert a paracrine effect on the adjacent nonproliferative cells therefore increasing the expression of the cell surface receptor [3].

Significant linear increase in the intensity of staining of EGFR in the differentiated cells of the stratum spinosum in oral leukoplakia with mild epithelial dysplasia has also been reported, which might be explained by the inducing role of TGF- $\alpha$ over DNA synthesis in noncycling cells [3].

These data not only support that increased dysregulation of epithelial cell growth is associated with increasing degrees of dysplasia, but are also suggestive of increased receptor-ligand interaction with increasing degrees of dysplasia.
Activation of the PI3K-AKT signal pathway may seems to be also closely related to oral precancerous lesions, with a genetic mutation being generated at a relatively early stage of cancer [4].

Additionally, PTEN, a dual protein/lipid phosphatase whose main substrate - phosphatidyl-inositol 3,4,5 triphosphate (PIP3) - is the product of PI3K, may be mutated or epigenetically inactivated in a large fraction of human tumors, rivaling with p53 as one of the most important tumor suppressor proteins. PTEN activity may be lost by mutations, deletions or promoter methylation silencing [5].

In oral cancer, genetic alterations in PTEN, located at 10q23.3, occur in $5-10 \%$ of oral squamous cell carcinoma lesions but, remarkably, loss of PTEN expression was observed by Lee et al. in up to $29 \%$ oral squamous cell carcinomas of the tongue. This lack of PTEN expression may be an independent prognostic indicator of poor clinical outcome [6].

Moreover, chronic inflammation has been known to induce neoplasia through the increased production of reactive oxygen and nitrogen species, which results in elevated DNA damage. Moreover, it may induce the expression of multiple tumor-promoting genes, such as the tumor necrosis factor gene (TNF), matrix metalloproteinases (MMP) genes, and vascular endothelial growth factor (VEGF) genes which contribute to enhanced cellular migration and angiogenesis through the regulation of the proinflammatory gene Nuclear Factor $\mathrm{kB}$ (NFkB) [7].

On the other hand, tumor-promoting phorbol esters induce COX2 gene expression by activating the protein-kinase C (PKC) pathway, with one of the downstream target of activated PKC being the AP-1 transcription factor complex [8].

Single nucleotide polymorphisms of COX-2 have also been associated with the risk of multiple cancers, with the first epidemiologic evidence indicating that potentially functional polymorphisms of the COX-2 gene may have an impact on individual susceptibility to oral premalignant lesions [9].

Furthermore, angiogenesis is crucial to the growth, invasion and metastasis of a tumour and a significant increase in vascularity occurs during the transition from normal oral mucosa, through differing degrees of dysplasia, to invasive squamous cell carcinoma.

Although studies involving the expression of VEGF dysplastic

*Corresponding author: Rui Amaral Mendes, Department of Oral Medicine/ Oncology and Clinical Pathology, Portuguese Catholic University, Portugal, Tel: +351-93-2805962; E-mail: ramaralmendes@crb.ucp.pt

Received December 18, 2012; Accepted December 19, 2012; Published December 28, 2012

Citation: Mendes RA (2012) Oncogenic Pathways in the Development of Oral Cancer. J Carcinogene Mutagene 3:133. doi:10.4172/2157-2518.1000133

Copyright: (c) 2012 Mendes RA. This is an open-access article distributed under the terms of the Creative Commons Attribution License, which permits unrestricted use, distribution, and reproduction in any medium, provided the original author and source are credited. 
Citation: Mendes RA (2012) Oncogenic Pathways in the Development of Oral Cancer. J Carcinogene Mutagene 3: e110. doi:10.4172/21572518.1000133

epithelium are somehow conflicting, VEGF expression was found to correlate with the degree of tumour differentiation, which is consistent with the results of previous studies, although the reason why the expression of VEGF appears to be reduced in poorly differentiated oral squamous cell carcinoma still remains unclear [10-12].

Interestingly, a study which assessed whether there were any gene amplifications ocurring in oral dysplasias and, most importantly, which were the oncogenic pathways disrupted in premalignancies, found out that gene amplification did occur in $40 \%$ of the early lesions but, most importantly, that affected genes were part of different canonical oncogenic pathways, of which they were able to identify 5 , which share common nodes and interact as a single network [13].

According to the histological model of oral carcinogenesis, cells chronically exposed to environmental carcinogens progress through the stages of reactive hyperkeratosis, epithelial hyperplasia, degrees of dysplasia and intraepithelial carcinoma leading to invasive carcinoma.

Comprehension of the underlying pathways governing the progression of oral premalignant lesions is of the utmost importance. The molecular changes antedate the occurrence of invasive malignancy and can be used for risk assessment and as intermediate endpoints for targeted therapies.

Focus on preinvasive stages of high-grade and low-grade lesions that are known to further develop into cancer will allow us to identify early genetic events during cancer development, as genetic alterations build up a neoplastic phenotype.

\section{Reference}

1. Ye Y, Lippman SM, Lee JJ, Chen M, Frazier ML, et al. (2008) Genetic variations in cell-cycle pathway and the risk of oral premalignant lesions. Cancer 113: 2488-2495.

2. Nagatsuka H, Ishiwari $Y$, Tsujigiwa $H$, Nakano K, Nagai N (2001) Quantitation of epidermal growth factor receptor gene amplification by competitive polymerase chain reaction in pre-malignant and malignant oral epithelial lesions. Ora Oncol 37: 599-604.

3. Srinivasan M, Jewell SD (2001) Evaluation of TGF-alpha and EGFR expression in oral leukoplakia and oral submucous fibrosis by quantitative immunohistochemistry. Oncology 61: 284-292.

4. Watanabe S, Sato K, Okazaki Y, Tonogi M, Tanaka Y, et al. (2009) Activation of PI3K-AKT pathway in oral epithelial dysplasia and early cancer of tongue. Bul Tokyo Dent Coll 50: 125-133.

5. Blanco-Aparicio C, Renner O, Leal JF, Carnero A (2007) PTEN, more than the AKT pathway. Carcinogenesis 28: 1379-1386.

6. Lee JI, Soria JC, Hassan KA, El-Naggar AK, Tang X, et al. (2001) Loss of PTEN expression as a prognostic marker for tongue cancer. Arch Otolaryngol Head Neck Surg 127: 1441-1445.

7. Nathan CO, Leskov IL, Lin M, Abreo FW, Shi R, et al. (2001) COX-2 expression in dysplasia of the head and neck: correlation with elF4E. Cancer 92: 18881895.

8. Yang SF, Chen MK, Hsieh YS, Chung TT, Hsieh YH, et al. (2010) Prostaglandin E2/EP1 signaling pathway enhances intercellular adhesion molecule 1 (ICAM1) expression and cell motility in oral cancer cells. J Biol Chem 285: 2980829816.

9. Pu X, Lippman SM, Yang H, Lee JJ, Wu X (2009) Cyclooxygenase-2 gene polymorphisms reduce the risk of oral premalignant lesions. Cancer 115: 14981506.

10. Molinolo AA, Amornphimoltham $\mathrm{P}$, Squarize $\mathrm{CH}$, Castilho RM, Patel V, et al (2009) Dysregulated molecular networks in head and neck carcinogenesis. Oral Oncol 45: 324-334.

11. Johnstone S, Logan RM (2006) The role of vascular endothelial growth factor (VEGF) in oral dysplasia and oral squamous cell carcinoma. Oral Oncol 42 337-42.

12. Johnstone S, Logan RM (2007) Expression of vascular endothelial growth factor (VEGF) in normal oral mucosa, oral dysplasia and oral squamous cel carcinoma. Int J Oral Maxillofac Surg 36: 263-266.

13. Tsui IF, Poh CF, Garnis C, Rosin MP, Zhang L, et al. (2009) Multiple pathways in the FGF signaling network are frequently deregulated by gene amplification in oral dysplasias. Int J Cancer 125: 2219-2228. 